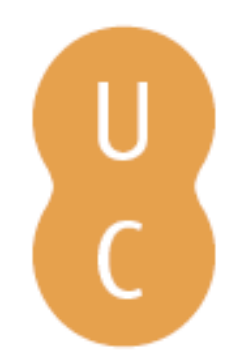

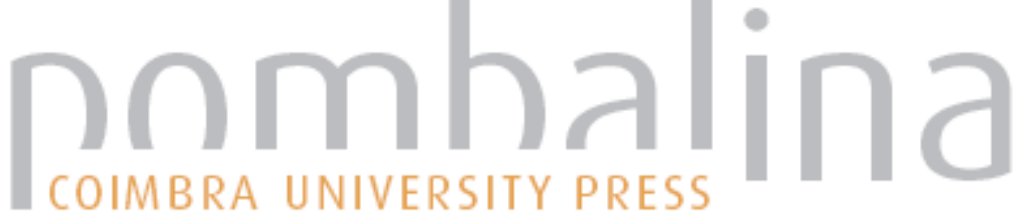

\section{Creating metamaterials at the nano-scale}

Autor(es): $\quad$ Pacheco, J. M.; Reis, C. L.

Publicado por: Imprensa da Universidade de Coimbra

URL

persistente: URI:http://hdl.handle.net/10316.2/31327

DOI: $\quad$ DOI:http://dx.doi.org/10.14195/978-989-26-0240-0_10

Accessed : $\quad$ 26-Apr-2023 10:33:05

A navegação consulta e descarregamento dos títulos inseridos nas Bibliotecas Digitais UC Digitalis, UC Pombalina e UC Impactum, pressupõem a aceitação plena e sem reservas dos Termos e Condições de Uso destas Bibliotecas Digitais, disponíveis em https://digitalis.uc.pt/pt-pt/termos.

Conforme exposto nos referidos Termos e Condições de Uso, o descarregamento de títulos de acesso restrito requer uma licença válida de autorização devendo o utilizador aceder ao(s) documento(s) a partir de um endereço de IP da instituição detentora da supramencionada licença.

Ao utilizador é apenas permitido o descarregamento para uso pessoal, pelo que o emprego do(s) título(s) descarregado(s) para outro fim, designadamente comercial, carece de autorização do respetivo autor ou editor da obra.

Na medida em que todas as obras da UC Digitalis se encontram protegidas pelo Código do Direito de Autor e Direitos Conexos e demais legislação aplicável, toda a cópia, parcial ou total, deste documento, nos casos em que é legalmente admitida, deverá conter ou fazer-se acompanhar por este aviso.

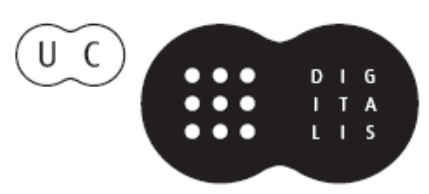


J. S. Redinha

J. da Providência

A. J. C. Varandas

Editors

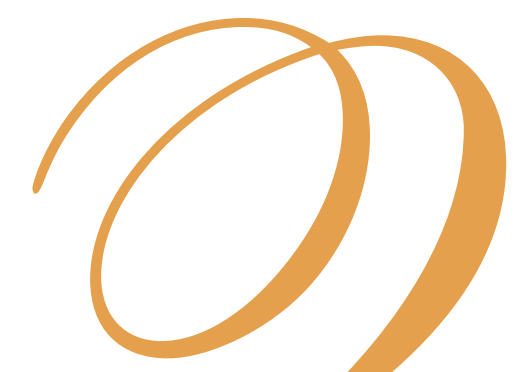

in Chemistry and Physics

A tribute to the memory of

Professor Couceiro da Costa

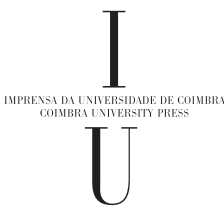




\title{
10. Creating Metamaterials at The NANO-SCAle
}

\author{
J. M. Pacheco ${ }^{1,2 *}$ and C. L. Reis ${ }^{1}$ \\ ${ }^{1}$ Departamento de Matemática e Aplicações, Universidade do Minho \\ 4710 - 057 Braga, Portugal \\ ${ }^{2}$ ATP-group, CMAF, Complexo Interdisciplinar da Universidade de Lisboa, \\ Av. Prof. Gama Pinto 2, 1649 - 003 Lisboa, Portugal
}

\begin{abstract}
The fact that the founding papers of Density Functional Theory are among the most cited papers ever, testifies for the importance of Quantum Mechanics and its (often) counter intuitive features in characterizing many-particle systems at a nano and sub-nano scale. Density Functional Theory has enabled one to use the computer to predict quantitatively several of the properties of the aforementioned many-particle systems. The prediction of new materials, often exhibiting meta-stability, is one of its distinctive features. In this lecture we will discuss a new class of meta-materials which, being silicon based, exhibit properties which in no way resemble those of its main constituent. In particular, we will discuss the structural and electronic properties of new materials of the form $X @ \mathrm{Si}_{16}$ (with $X=\mathrm{Ti}, \mathrm{Zr}$ and $\mathrm{Hf}$ ) which are predicted to be (meta) stable at room temperature and exhibit a remarkable potential as possible high- $T_{c}$ superconductors.
\end{abstract}

\subsection{Introduction}

A fundamental challenge for nanotechnology is to control fabrication with atomic precision in order to assemble new materials with outstanding properties or functions. Modern theoretical and computational methods are already able to predict the properties of such materials. The importance of this has been recognized with the 1998 Nobel Prize award to Walter Kohn for his development of the Density Functional Theory [1,2] (DFT) and to John Pople for his development of computational methods in quantum theory. Today, theoretical predictions are competitive with experimental techniques for controlling single

\footnotetext{
"Email address: jmpacheco@math.uminho.pt
} 
molecule chemistry given that the required hardware for performing computer simulations is often orders of magnitude cheaper and may be more effective than experiments. The quantitative predictions based on DFT rely on Quantum Mechanics which itself is on the basis of the technological revolution of the $\mathrm{XX}^{\text {th }}$ century.

Much of what is considered now the information technology revolution has been dependent of an ever increasing miniaturization of devices based on silicon. The number of transistors that can be placed in an integrated circuit, which is a measure of its computing power, has doubled every 20 months since 1971. However, physical limits to miniaturization of devices based on bulk silicon have already been met in the recent $45 \mathrm{~nm}$ generation of devices, where a high$\mathrm{k}$ dielectric material like $\mathrm{HfO}_{2}$ has replaced $\mathrm{SiO}_{2}$ as a gate insulator [3] for the first time since the beginning of the integrated circuit. The potential "brick wall" facing Moore's Law [4] has motivated an incredible amount of experimental and theoretical work in the search for alternative materials to bulk silicon. Silicon clusters in particular have been under focus, given that nano-structured materials are known to exhibit very different properties from their bulk counterparts. But contrary to fullerene-like carbon clusters, pure silicon clusters have been found to be chemically reactive, precluding the synthesis of cluster assembled materials [5]. Along another route, early experiments by Beck [6,7] indicated the feasibility of using metal atoms to nucleate several silicon atoms into stable $X @ S i_{n}$ clusters, of which $X @ S i_{16}$ was found to be particularly stable. Recent experimental [5,8-15] and theoretical [16-24] work has confirmed these results for a variety of mixed metal-silicon sandwich [8,25] and cage [16, 17, 23, 24] clusters, and a special class of clusters with stoichiometry $X @ S_{16}$, with $X$ a metal atom, has been identified [16] as especially stable by means of ab-initio computer simulations. In particular, the stability of $X @ S_{16}(X=\mathrm{Ti}, \mathrm{Zr}, \mathrm{Hf})$ nano particles has been confirmed experimentally [13], via selective formation of neutral gas phase clusters, using a dual laser vaporisation technique of pure metal and pure silicon targets in an inert helium atmosphere. An additional experimental confirmation of the synthesis of these nano-particles has been reported recently using a mag- 
netron co-sputtering tecnhique [15].

Using first-principles computer simulations within DFT we investigate the main electronic properties of $X @ S_{16}(X=\mathrm{Ti}, \mathrm{Zr}$ and Hf $)$ clusters. The vibrational modes and infrared spectra are also determined. We show the feasibility of using the clusters as elementary building blocks to synthesize stable bulk materials, and find that all the $X @ \mathrm{Si}_{16}(X=\mathrm{Ti}, \mathrm{Zr}$ and Hf) cluster-assembled materials crystallize in hexagonal closed packed structures (HCP). We further characterize the main structural and electronic properties of these materials, while illustrating their differences. We predict that these materials should be possible to stabilize in a metastable phase at room temperature and normal pressure conditions. This phase is predicted to be maintained under isotropic compression up to $\sim 1 \mathrm{GPa}$. Similar to Ti@Si 16 , both $\mathrm{Zr@Si}{ }_{16}$ and $\mathrm{Hf} @ \mathrm{Si}_{16}$ are especially stable semiconductors with GGA (see below) band gaps of $1.6 \mathrm{eV}, 0.3 \mathrm{eV}$ larger than that previously found for bulk Ti@Si 16 .

This paper is organized as follows: In section two details of the method and simulations carried out are provided. Results and discussion are left to section three, whereas the main conclusions and future prospects are postponed to section four.

\subsection{Methods}

All ab-initio calculations were performed within the generalized gradient approximation (GGA [27]) to DFT using norm-conserving pseudopotentials $[26,28]$ and a plane-wave basis $[29,30]$. An energy cut-off of 30.0 Hartree (816 eV) was used throughout, leading to well converged forces within $0.02 \mathrm{eV} /$ Bohr. This value was also used as a stopping criteria for structural optimizations. Large energy cutoffs are crucial to ensure reliable results (and good convergence of the forces). We note that if less restrictive (and consequently, less computer demanding) parameters are used in structural optimizations, the forces (gradients of the energy with respect to atomic positions) will be poorly determined. As a consequence artificial structures and cage breakup can be obtained using $X @ \mathrm{Si}_{16}$ clusters as building blocks. 


\subsubsection{Isolated Clusters}

\section{Structural optimization}

The atomic coordinates of the isolated clusters were computed employing a super-cell hexagonal lattice with parameters $a=c=27.0$ Bohr to avoid mirrorimage interactions. To ensure proper structure determination we performed several Quantum Langevin Molecular Dynamics [31] (QLMD) simulations at different temperatures starting from arbitrary configurations of Si atoms always nucleated around the central metal atom. Subsequently we performed geometry optimizations employing a conjugated gradient algorithm starting from the lowest energy configurations obtained in the QLMD runs.

\section{Electronic properties}

We computed the total energy, the one-electron Kohn-Sham levels as well as the total valence electronic density $\rho(\boldsymbol{r})$ of each nano-structure at the equilibrium configuration. From the electronic density $\rho(\boldsymbol{r})$ we constructed the radial electronic density, $\rho(r)=\rho(|\boldsymbol{r}|)$ by calculating its average over the solid angle:

$$
\rho(r)=\frac{1}{4 \pi} \int_{\Omega} \rho(\boldsymbol{r}) \mathrm{d} \Omega
$$

The number of valence electrons is given by:

$$
N=\int \mathrm{d}^{3} r \rho(\boldsymbol{r})=\int_{0}^{\infty} \mathrm{d} r 4 \pi r^{2} \rho(r) \equiv \int_{0}^{\infty} \mathrm{d} r \eta(r)
$$

The quantity $\eta(r)$ defined in the last integral can be useful in quantifying the electronic density inside the nanoparticle, providing a qualitative measure of its chemical inertia.

We computed the cohesive energy per atom for each cluster subtracting from the total energy $E_{\mathrm{tot}}$ the atomic energies $E_{\mathrm{ps}}^{\mathrm{Si}}$ and $E_{\mathrm{ps}}^{X}(X=\mathrm{Ti}, \mathrm{Zr}, \mathrm{Hf})$ of the pseudopotential calculation, $E_{\mathrm{coh}}=\left(E_{\mathrm{tot}}-16 E_{\mathrm{ps}}^{\mathrm{Si}}-E_{\mathrm{ps}}^{X}\right) / 17$.

\section{Vibrational modes and infrared spectrum}

The vibrational modes of frequency $\omega$ are described by a periodic displacement in time of each nuclei $I$ :

$$
\mathbf{u}_{I}(t)=\mathbf{u}_{I} e^{i \omega t}
$$


This leads to the following eigenvalue equation:

$$
-\omega^{2} M_{I} \mathbf{u}_{I}=\sum_{J} \frac{\partial^{2} E(\mathbf{R})}{\partial \mathbf{R}_{I} \partial \mathbf{R}_{J}} \mathbf{u}_{J}
$$

which involves second order derivatives of the ground state energy $E(\mathbf{R})$ with respect to all $N$ nuclei positions $\mathbf{R}_{I}(I=1, \ldots, N)$. Solving these equations leads to a set of frequencies $\omega^{\nu}(\nu=1, \ldots, 3 N)$ and corresponding normal modes $\mathbf{u}^{\nu}=\mathbf{u}_{\tau, \alpha}^{\nu} \mathbf{e}_{\alpha}$ involving the collective displacements of the nuclei $(\tau=1, \ldots, N)$ along the Cartesian directions $(\alpha=x, y, z)$.

The absolute infrared intensity of the mode $\nu$ is given by [32]:

$$
I_{\nu}^{\mathrm{IR}}=K\left|\sum_{\tau, \alpha, \beta} Z_{\tau, \alpha, \beta}^{*} u_{\tau, \beta}^{\nu}\right|^{2}
$$

with $\tau=1, \ldots, N$ and $\alpha, \beta=x, y, z$. For intensities in $(\mathrm{D} / \AA)^{2} \mathrm{amu}^{-1}$ and $Z$ in atomic units $K=4.2056 \times 10^{4}$. The Born effective charge tensor $\mathbf{Z}^{*}$ is the second derivative of the energy with respect to both the electric field $\mathbf{G}$ and the nuclei displacement $\mathbf{R}_{\tau}$ :

$$
Z_{\tau, \alpha, \beta}^{*}=\frac{\partial^{2} E}{\partial G_{\alpha} \partial R_{\tau, \beta}}
$$

The second order derivatives of the ground state energy, with respect to atomic displacements and/or homogeneous electric fields are computed using density functional perturbation-theory (DFPT) $[33,34]$.

\subsubsection{Bulk phase}

\section{Structural optimization}

In a first step, we investigate bulk forms of cluster assembled materials, using the equilibrium structures of the isolated cages. To this end, we computed the cohesive energy per cluster varying the distance between clusters in a given bulk structure, while freezing the cluster geometry and the angles between primitive lattice vectors. The cohesive energy per cage in the bulk $E_{\mathrm{coh}}^{b}$ is defined:

$$
E_{\mathrm{coh}}^{b}=\left(E_{\mathrm{tot}}^{b}-N_{c} E_{I}\right) / N_{c}
$$

where $E_{\text {tot }}^{b}$ is the total energy per untit cell, $E_{I}$ is the energy of the isolated cluster and $N_{c}$ is the number of clusters in the unit cell. Several bulk structures 
were investigated: Simple Cubic (SC), DIAmond-type (DIA), Body Centered Cubic (BCC), Face Centered Cubic (FCC) and Hexagonal Close Packed (HCP). We placed 1 cluster per unit cell in the SC, BCC and FCC structures, and 2 in DIA and HCP. We have also tried to use supercells for the different lattices but the corrections in energy were found to be negligible. We have carefully chosen the $k$-point sampling in each calculation (particularly for small inter-cluster distances) in order to ensure well converged results. We used the following Monkhorst-Pack grids: $2 \times 2 \times 2$ for DIA, $4 \times 4 \times 4$ for SC, BCC and FCC and $3 \times 3 \times 2$ for HCP.

Subsequently we performed a full geometry relaxation of both atomic coordinates and lattice parameters taking as a starting point the configuration corresponding to the minimum of the cohesive energy per cluster as a function of distance between clusters for the different bulk structures we found before.

\section{Pressure curve and bulk modulus}

Given the cohesive energy per cluster as a function of the distance $d$ between clusters, $E_{\mathrm{coh}}(d)$, we can obtain the pressure as a function of inter-cage distance $P(d)$ by computing the numerical derivative from a cubic spline fit to the cohesive energy points:

$$
P(d)=-\frac{\partial E}{\partial V}=-\frac{\partial E}{\partial d}\left(\frac{\partial V}{\partial d}\right)^{-1}
$$

For a hexagonal lattice in the ideal packing structure (HCP), the volume of the primitive cell is $V=\sqrt{2} d^{3}$. Thus:

$$
P(d)=-\frac{1}{3 \sqrt{2} d^{2}} \frac{\partial E}{\partial d}=-\frac{254.845}{d^{2}} \frac{\partial E}{\partial d}
$$

which provides the pressure in GPa for lengths in Bohr and energies in $\mathrm{eV}$. The Bulk modulus $B$ is determined by fitting the cohesive energy points to the Birch-Murnaghan equation of state [35]:

$$
E(V)=E_{0}+\frac{9 V_{0} B_{0}}{16}\left\{\left[\left(\frac{V_{0}}{V}\right)^{2 / 3}-1\right]^{3} B_{0}^{\prime}+\left[\left(\frac{V_{0}}{V}\right)^{2 / 3}-1\right]^{2}\left[6-4\left(\frac{V_{0}}{V}\right)^{2 / 3}\right]\right\}
$$



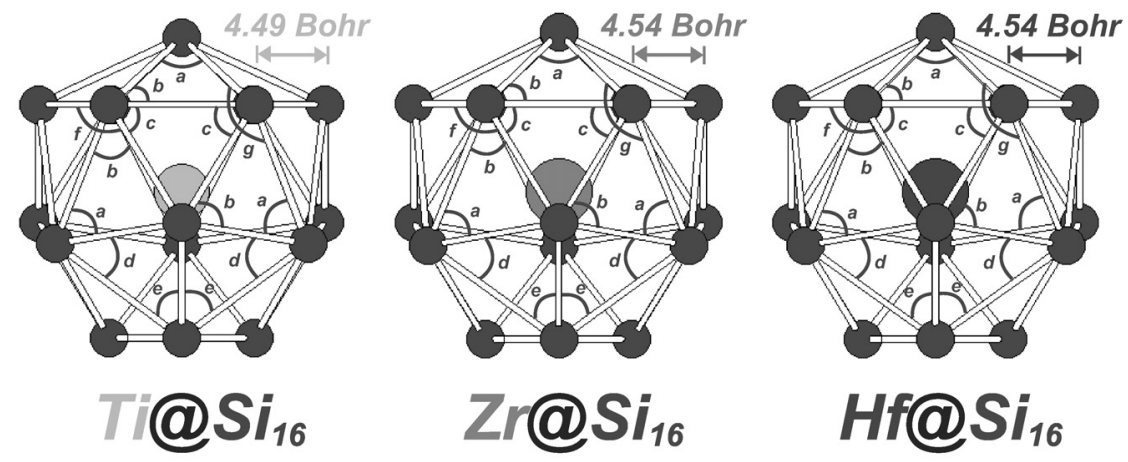

\section{$H f @ S i_{16}$}

Figure 10.1. The Frank-Kasper [36] cage-structures, corresponding to the equilibrium of the $X @ S_{16}$ nano-particles. These highly symmetric structures, exhibiting several $C_{3}$ symmetry axes, will be used as building blocks of molecular solids. Selected bond angles are also represented. Angle values are given in Table 10.1.

Table 10.1. Selected bond angles depicted in Figure 10.1 for the $X @ S_{16}$ clusters $(X=\mathrm{Ti}$, $\mathrm{Zr}$, Hf). Similar values for the angles have been identified in amorphous Silicon [37].

\begin{tabular}{lccccccc}
\hline$X @ \mathrm{Si}_{16}$ & $a$ & $b$ & $c$ & $d$ & $e$ & $f$ & $g$ \\
\hline $\mathrm{Ti}$ & $54.6^{\circ}$ & $62.7^{\circ}$ & $60.0^{\circ}$ & $53.1^{\circ}$ & $63.4^{\circ}$ & $120.0^{\circ}$ & $106.4^{\circ}$ \\
$\mathrm{Zr}$ & $54.0^{\circ}$ & $63.0^{\circ}$ & $60.0^{\circ}$ & $52.6^{\circ}$ & $63.7^{\circ}$ & $120.0^{\circ}$ & $108.4^{\circ}$ \\
$\mathrm{Hf}$ & $54.0^{\circ}$ & $63.0^{\circ}$ & $60.0^{\circ}$ & $52.6^{\circ}$ & $63.7^{\circ}$ & $120.0^{\circ}$ & $108.4^{\circ}$ \\
\hline
\end{tabular}

\subsection{Results and Discussion}

\subsubsection{Isolated Clusters}

The structures of the isolated $X @ S_{16}$ nano-particles obtained using the procedure outlined in the previous section are shown in Figure 10.1.

All these nano-particles exhibit Frank-Kasper [36] cage structures with $C_{3 v}$ symmetry. In Table 10.2 the parameters characterizing the structural properties of these clusters are given. We choose three sets of distances: The distance from the metal atom to the four silicon atoms on the tetrahedral sites $r_{1}$; the distance from the metal atom to the remaining twelve silicon atoms $r_{2}$, and the minimum nearest neighbour Si - Si distance $r_{\text {min }}^{n n}$. The $\mathrm{Zr@Si} 16$ and $\mathrm{Hf} @ \mathrm{Si}_{16}$ have larger dimensions than Ti@Si $\mathrm{S}_{16}: r_{1}$ and $r_{2}$ are $\sim 3 \%$ and $\sim 1 \%$ larger than the ones found for Ti@Si $i_{16}$.

Once the ground state geometries have been determined, we computed their 
Table 10.2. Structural parameters for the $X @ \mathrm{Si}_{16}$ clusters with $X=\mathrm{Ti}, \mathrm{Zr}$, Hf. $r_{1}$ is the distance from the metal atom to the four silicon atoms on the tetrahedral sites, $r_{2}$ is the distance of the metal atom to the remaining twelve silicon atoms and $r_{\min }^{n n}$ is the minimum nearest neighbour Si-Si distance.

\begin{tabular}{lccc}
\hline$X @ \mathrm{Si}_{16}$ & $r_{1}(\mathrm{Bohr})$ & $r_{2}(\mathrm{Bohr})$ & $r_{\text {min }}^{\text {nn }}$ \\
\hline $\mathrm{Ti}$ & 4.93 & 5.34 & 4.49 \\
$\mathrm{Zr}$ & 5.09 & 5.40 & 4.54 \\
$\mathrm{Hf}$ & 5.09 & 5.40 & 4.54 \\
\hline
\end{tabular}

Table 10.3. Cohesive energy per cluster and HOMO-LUMO gaps for the $X @ \mathrm{Si}_{16}$ clusters with $X=\mathrm{Ti}, \mathrm{Zr}$, Hf.

\begin{tabular}{lcc}
\hline$X @ \mathrm{Si}_{16}$ & $E_{\text {coh }} /$ Atom $(\mathrm{eV})$ & $H-L \mathrm{Gap}(\mathrm{eV})$ \\
\hline $\mathrm{Ti}$ & -4.96 & 2.3 \\
$\mathrm{Zr}$ & -4.99 & 2.4 \\
$\mathrm{Hf}$ & -4.97 & 2.5 \\
\hline
\end{tabular}

main electronic properties. In Table 10.3 we list the calculated cohesive energy per atom and HOMO-LUMO (Highest Occupied - Lowest Unoccupied Molecular Orbital) gap for these clusters. Whereas the cohesive energies are almost identical for all cages the $\mathrm{Zr@Si}_{16}$ and $\mathrm{Hf@Si} 16$ gaps are $~ 6 \%$ larger than the one found for $\mathrm{Ti} @ \mathrm{Si}_{16}$.

In Figure 10.2 we show the radial electronic density of all the three clusters, which is remarkably similar. Besides their large HOMO-LUMO gaps, another indication of stability in these clusters is that almost all of the electronic density is concentrated inside the cage clusters. The vertical bars represent the outer limits of the cage-cluster, taking into account the cage radius and the silicon atomic radius (cf. Table 10.2). Approximately 96\% of the electronic charge density is concentrated inside a sphere of radius 8 Bohr, suggesting a remarkable level of chemical inertia.

In Figure 10.3 we display the one-electron energy levels. The three nanoparticles exhibit energy level distributions which are qualitatively similar. The degeneracies of the energy levels can be qualitatively organized in the following 


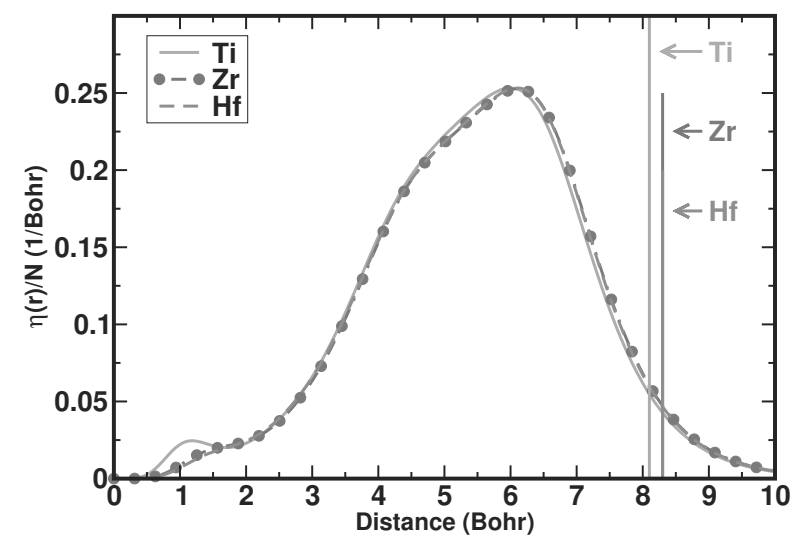

Figure 10.2. Normalized radial electronic densities $\eta(r)=4 \pi r^{2} \rho(r)$ plotted as a function of the distance to the central metal atom for isolated clusters Ti@Si $i_{16}$ (solid line), Zr@Si 16

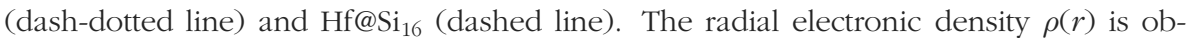
tained from the calculated ground state total electronic density $\rho(\boldsymbol{r})$ taking its average over the solid angle $\Omega$. See Eq. (10.1). The total area subtended by each curve is 1 .

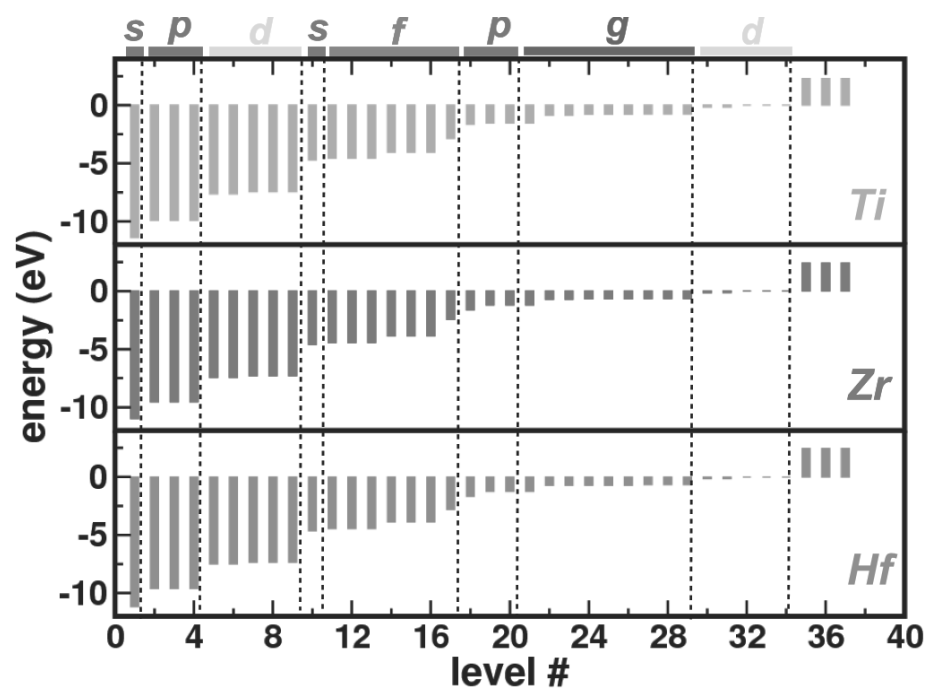

Figure 10.3. Energy levels for the $X @ \mathrm{Si}_{16}$ clusters with $X=\mathrm{Ti}, \mathrm{Zr}$, Hf. The electronic occupancy of each level is 2 . The plotted levels are grouped according to their approximated degeneracies corresponding to levels in a spherical (jellium) shell structure with angular momenta $s, p, d, s, f, p, g, d$. 


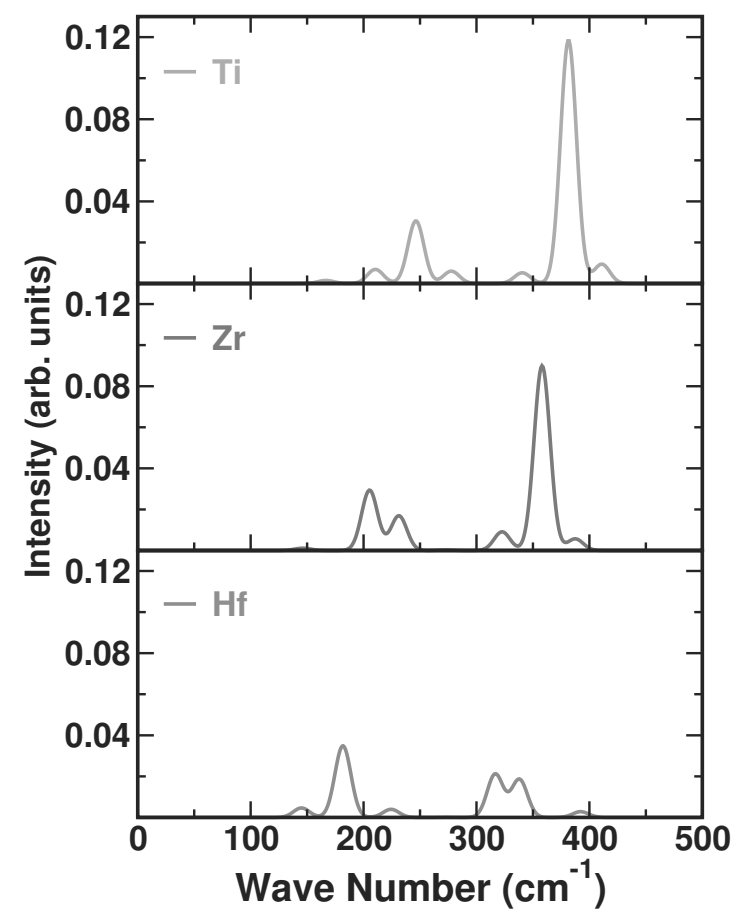

Figure 10.4. The calculated infrared spectrum of the $X @ \mathrm{Si}_{16}(X=\mathrm{Ti}, \mathrm{Zr}$ and Hf) nanoparticles.

sequence:

$$
2,6,10,2,14,6,18,10
$$

This sequence is in excellent agreement with that resulting from a spherical-like (jellium) super-atom:

$$
s, p, d, s, f, p, g, d \text {. }
$$

Hence, and on top of a structurally stable and highly symmetric cluster, the 68 valence electrons of each cage cluster also organize into into a spherical closedshell electronic system. Consequently these cages qualify as "double magic".

In Figure 10.4 we show the results of the calculation of the infrared spectrum using the procedure described in the previous section. In all three cases the normal modes have frequencies which are low compared to the fullerenes, exhibiting sizable intensity $\sim 200 \mathrm{~cm}^{-1}$. This picture is consistent with a weaker bonding of the silicon atoms in the $X @ S_{16}(X=\mathrm{Ti}, \mathrm{Zr}$ and $\mathrm{Hf})$ nanoparticles 


\section{Ti}
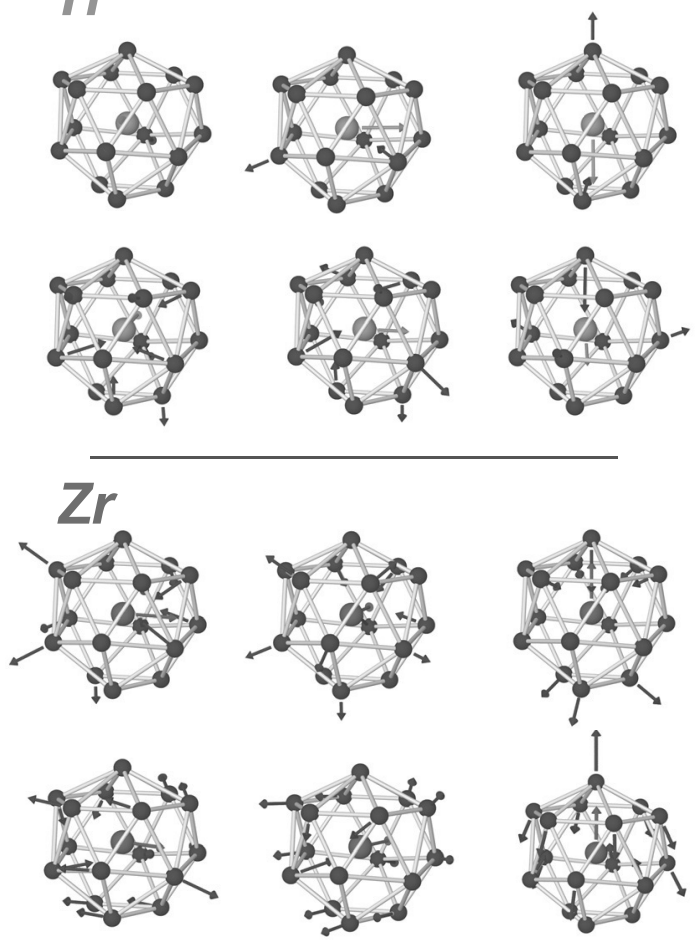

Figure 10.5. Selected normal modes for the Ti@Si ${ }_{16}$ (uppper panel) and $\mathrm{Zr@Si}_{16}$ (lower panel) nanoparticles. In each panel the three top modes correspond to the peak of highest IR activity whereas the lower three modes correspond to second most intense peak.

compared to the carbon atoms in the fullerene clusters. The IR spectrum for Ti@Si ${ }_{16}$ depicted in the upper panel of Figure 10.4 is nearly identical to the one obtained by Nakajima and coworkers [14] using a localized basis set method. One interesting feature apparent in Figure 10.4 is a progressive softening of the frequency spectrum with the increasing mass of the central metal atom. This is accompanied with a simultaneous decrease in IR activity which is most pronounced for Hf@Si $i_{16}$. The peaks of highest intensity at $380 \mathrm{~cm}^{-1}$ and $360 \mathrm{~cm}^{-1}$ for $\mathrm{Ti} @ \mathrm{Si}_{16}$ and $\mathrm{Zr} @ \mathrm{Si}_{16}$ respectively correspond to the three normal modes depicted in the upper part of each panel of Figure 10.5. In the case of Ti@Si $i_{16}$ these are essentially displacements of the central metal atom in the cluster with minor rearrangements of the surrounding silicon atoms. For the $\mathrm{Zr@Si}_{16}$ the 


\section{Hf}
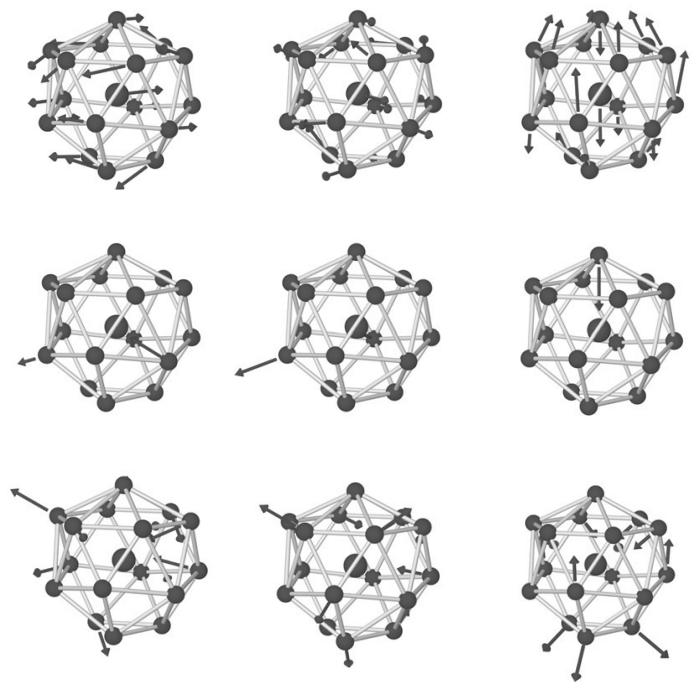

Figure 10.6. Selected normal modes for the Hf@Si ${ }_{16}$ nanoparticles. The three top modes correspond to the peak of highest IR activity whereas the lower six modes correspond to second most intense peaks.

movement of the central metal atom is accompanied by a more sizable distortion of the silicon cage. This is also the case for the three modes corresponding to the second most intense peak for both Ti@Si $i_{16}$ and $\mathrm{Zr@Si} 16$ at $246 \mathrm{~cm}^{-1}$ and $205 \mathrm{~cm}^{-1}$ respectively.

In the case of Hf@Si ${ }_{16}$ the peak of highest IR activity is located at $180 \mathrm{~cm}^{-1}$. These modes are depicted in the upper part of Figure 10.6 whereas the second most intense peaks correspond to the six normal modes depicted in the lower part of Figure 10.6.

\subsubsection{Bulk phase}

We investigate now the possible stability of bulk forms of the cluster assembled materials. We restrict our analysis to the Frank-Kasper [36] cage structures even though we are aware that other isomers of $M @ \mathrm{Si}_{16}$ have been reported in the literature $[16,21,22]$. However no structure of comparable stability 


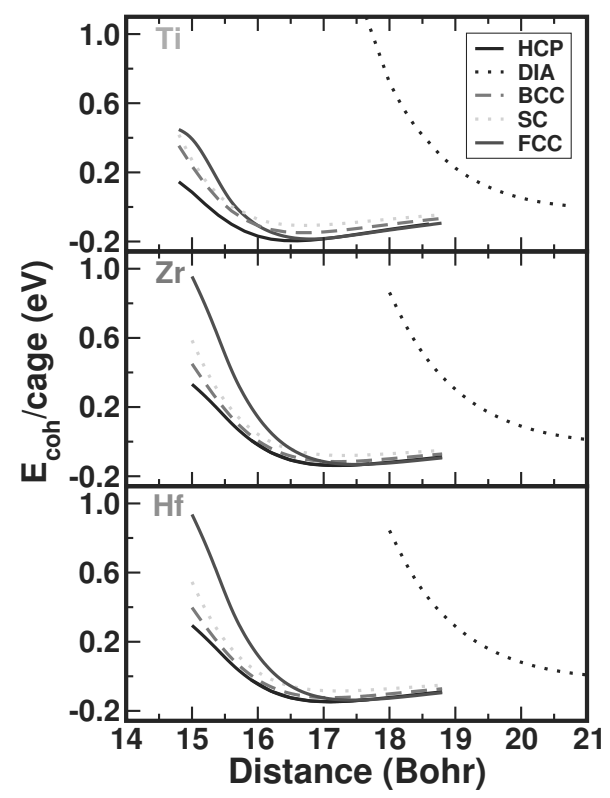

Figure 10.7. Cohesive energy as a function of inter-cage distance for bulk structures of $X @ \mathrm{Si}_{16}$ clusters with $X=\mathrm{Ti}, \mathrm{Zr}$, Hf. For all crystal structures, nearest neighbor cages are all at the same distance from any focal cage (for the HCP structure $(c / a=\sqrt{8 / 3}$, see main text for details). The curves for the HCP, FCC, BCC and SC are drawn with solid lower, solid upper, dashed and dotted lines, respectively. The only curve which exhibits no bound state corresponds to the DIA structure, drawn with a dotted line.

has been identified to date with stoichiometry $M @ S_{16}$. Hence we believe this choice is justified. The existence of a $C_{3 v}$ axis in the Frank-Kasper [36] structure may favour the HCP structure, since $C_{3 v}$ is the point symmetry group of the crystallographic $P 3 m 1$ hexagonal group; nonetheless we investigated other possibilities. In Figure 10.7 we plot the cohesive energy per cluster as a function of cage-cage distance for the three cluster assembled materials in their different bulk structures - SC, DIA, BCC, FCC and HCP. In all cases, the cohesive energy curves for the SC, BCC, and FCC structures exhibit well defined minima around 17 Bohr. They are, however, less stable than the HCP structure. An entirely different behaviour is found for the DIAmond structure indicating that in all cases this structure is unstable. In Figure 10.8 we show in detail the cohesive energy 


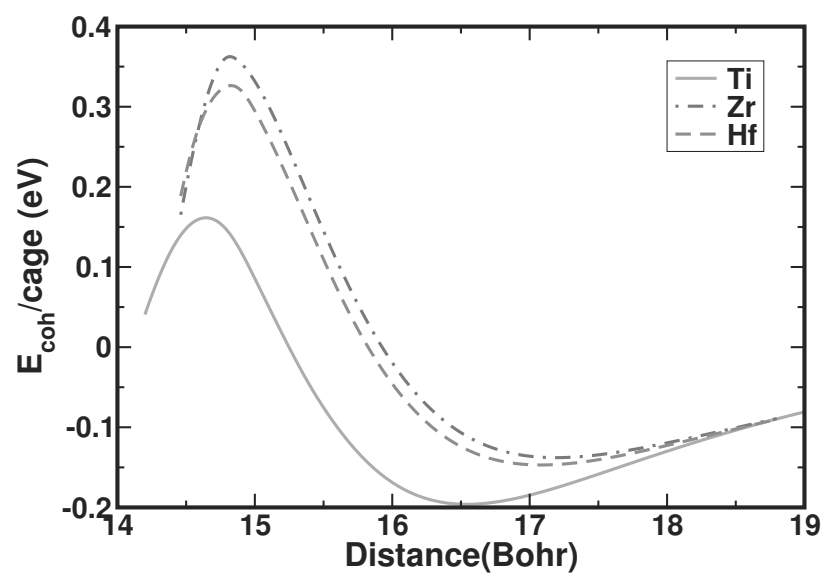

Figure 10.8. Cohesive energy as a function of inter-cage distance for the HCP molecular solids Ti@Si $i_{16}$ (solid line), Zr@Si ${ }_{16}$ (dashed-dotted line) and Hf@Si ${ }_{16}$ (dashed line).

curves for the three cluster assembled materials in the HCP structure. The curve for HCP-Ti@Si ${ }_{16}$ has a minimum for a cage-cage distance of 16.54 Bohr and a value at the minimum of only $-0.2 \mathrm{eV}$ indicating that the cages bind weakly. The significant reduction of the binding compared to fullerite [38] (cohesive energy per cluster of $-1.6 \mathrm{eV}$ ) is related to the role played by the central metal atom which effectively pulls the valence charge density to within the cage, increasing not only the cluster structural stability but also the HOMO-LUMO gap therefore

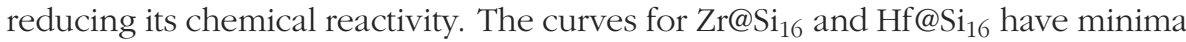
at cage-cage distances of 17.2 Bohr and 17.1 Bohr respectively. The inter-cage distance in these two structures is $\sim 4 \%$ larger than the one found for HCP Ti@Si $i_{16}$. The values of $-0.14 \mathrm{eV}$ and $-0.15 \mathrm{eV}$ at the minimum also indicate that the binding in these bulk materials is weaker than in the bulk Ti@Si ${ }_{16}$. These

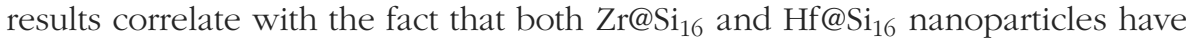
a cage radius $\sim 3 \%$ larger than $\mathrm{Ti}_{\mathrm{S}} \mathrm{Si}_{16}$. Indeed, a larger cage radius induces an increase of the inter-cage distance for the cluster assembled materials and also a decrease of the binding between clusters given that the same electronic charge is spread in a larger cluster volume. 
Table 10.4. Lattice parameters for the $X @ \mathrm{Si}_{16} \mathrm{HCP}$ molecular solids with $X=\mathrm{Ti}$, Zr, Hf. $\Delta$ is the deviation of the ratio of lattice parameters $c / a$ from the ideal packing value $\sqrt{8 / 3}$.

\begin{tabular}{lccc}
\hline$X @ \mathrm{Si}_{16}$ & $a$ (Bohr) & $c$ (Bohr) & $\Delta(\%)$ \\
\hline $\mathrm{Ti}$ & 16.54 & 27.13 & 0.5 \\
$\mathrm{Zr}$ & 17.11 & 27.94 & 0.01 \\
$\mathrm{Hf}$ & 16.93 & 28.14 & 1.8 \\
\hline
\end{tabular}

Relaxation of both the internal cluster coordinates and the lattice parameters starting at the minimum structures of Figure 10.8 leads to HCP structures characterized by the lattice parameters summarized in Table 10.4. The atomic rearrangements within each cluster are negligible compared to the isolated cluster geometry, the same applying to the overall changes in cohesive energies. The

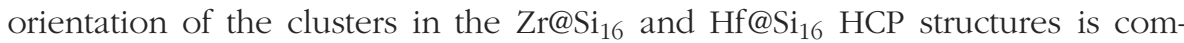
patible with the $p 3 m 1$ crystallographic group and identical to that of $\mathrm{Ti}_{\mathrm{S}} @ \mathrm{Si}_{16}$ in Ref. 26 where it has been explicitly illustrated.

Figure 10.8 also reveals that, despite the well developed minima in the cohesive energy per cluster, these minima are separated by barriers from other equilibrium structures [26], which turn out to be more stable.

Similar to what was found for Ti@Si ${ }_{16}$ [26] these systems will relax to an amorphous structure where silicon atoms of neighbouring cages bind covalently when subject, e.g., to very high pressures. This covalent binding leads to an absolute increase of the cohesive energy per cluster to $-2.2 \mathrm{eV}$. However from Figure 10.8 it is apparent that the values of the barrier maxima for both $\mathrm{Zr} @ \mathrm{Si}_{16}$ and $\mathrm{Hf} @ \mathrm{Si}_{16}$ are larger than the $0.16 \mathrm{eV}$ found for Ti@Si $\mathrm{I}_{16}$. This translates into an increase in the applied pressure necessary to drive the $\mathrm{Zr}$, Hf@Si ${ }_{16}$ bulk materials away from their metastable equilibrium HCP structure. Fully unconstrained geometry relaxations, varying both the cluster coordinates and unit cell parameters, starting from a configuration significantly compressed with respect to the equilibrium HCP configuration show no sign of amorphous transition at normal temperature.

In Figure 10.9 we plot the pressure as a function of inter-cage distance for the three $X @ \mathrm{Si}_{16}, X=\mathrm{Ti}, \mathrm{Zr}$, Hf bulk materials using the data from the cohesive 


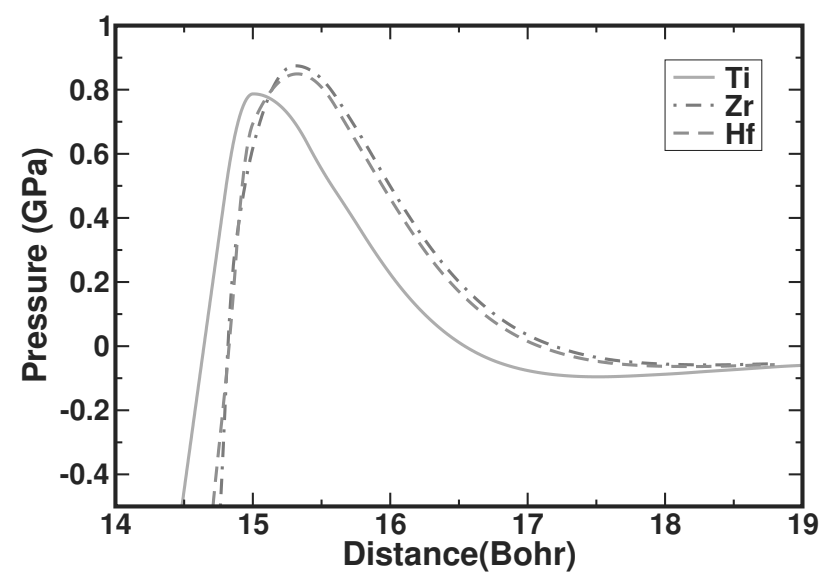

Figure 10.9. Computed pressure as a function of inter-cage distance for the HCP molecular solids Ti@Si 16 (solid line), Zr@Si $\mathrm{Si}_{16}$ (dash-dotted line) and Hf@Si $\mathrm{H}_{16}$ (dashed line). The curves were obtained by computing the numerical derivative of the cubic spline fit to the cohesive energy points used to plot Figure 10.8. See Eq. (10.2).

Table 10.5. Bulk modulus for the X@Si ${ }_{16}$ HCP molecular solids with $X=\mathrm{Ti}, \mathrm{Zr}$, Hf.

\begin{tabular}{cc}
\hline$X @ \mathrm{Si}_{16}$ & Bulk Modulus (GPa) \\
\hline $\mathrm{Ti}$ & 1.25 \\
$\mathrm{Zr}$ & 0.90 \\
$\mathrm{Hf}$ & 0.97 \\
\hline
\end{tabular}

energy curves and Eq. (10.2). We found that the maxima of the pressure curves are $0.87 \mathrm{GPa}$ for bulk $\mathrm{Zr@Si}{ }_{16}$ and 0.85 GPa for bulk Hf@Si ${ }_{16}$, values 8\% larger than the 0.79GPa obtained for the bulk Ti@Si ${ }_{16}$ indicating that both bulk Zr@Si $i_{16}$ and Hf@Si $\mathrm{S}_{16}$ are more stable than bulk Ti@Si 16 against applied pressure. The values for the bulk modulus $B$, obtained by fitting the Birch-Murnaghan equation of state, Eq. (10.3), to the cohesive energy points are given in Table 10.5.

Quantum Langevin molecular dynamics (QLMD) simulations [31], starting at the Ti@Si ${ }_{16}$ HCP equilibrium structure, suggest that the HCP phase is probably stable at room temperature, as shown in Figure 10.10. QLMD simulations provide a very efficient test of the overall stability of the system, given the feasibility of observing the occurrence of structural phase transitions, whenever they 


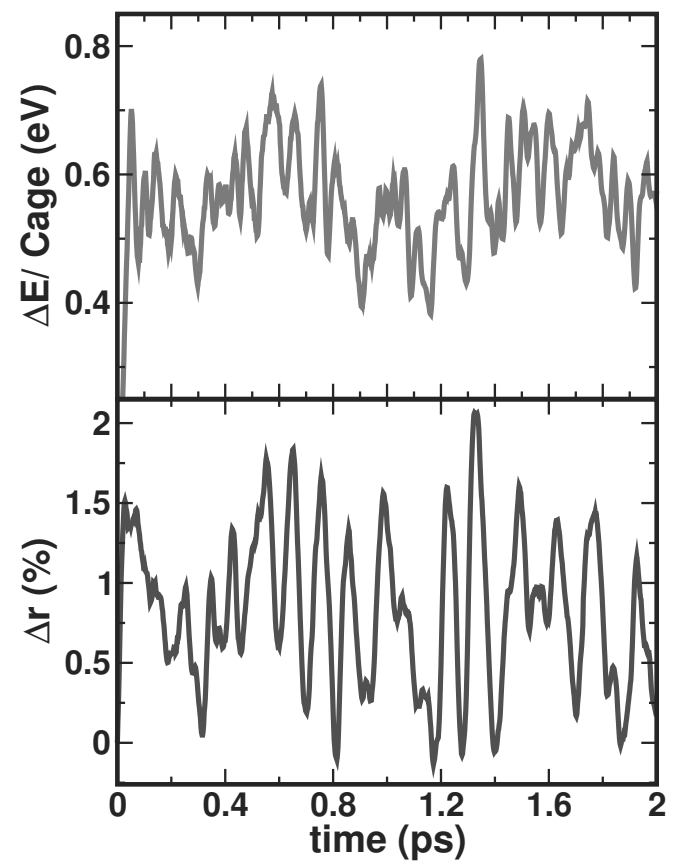

Figure 10.10. Upper Panel: Change of total energy per cage (with respect to equilibrium HCP configuration) as a function of time for a variable cell-shape quantum Langevin molecular dynamics of bulk-Ti@Si ${ }_{16}$. Simulation started from the HCP structure at a temperature of $300 \mathrm{~K}$. Lower Panel: Time dependence of the percentual deviation (with respect to the equilibrium value) of the average radius of each cage. The results show the small amplitude of the oscillations taking place at room temperature, and suggest the probable stability of this material. The time step used in each iteration is $2 \times 10^{-15} \mathrm{sec}$, and the simulation ran for a total of $2 \times 10^{-12} \mathrm{sec}$.

actually take place. QLMD combines some of the advantages of Metropolis Monte Carlo (MC) and MD simulations. By exploiting the energy gradient the atoms move collectively to the minima thereby efficiently sampling the configuration space. This is generally more efficient than a MC procedure where the position of a single atom is updated at each step, followed by a recalculation of the energy. The evaluation of the gradients of the energy, i.e., atomic forces and stress are performed at almost no cost once the energy is determined.

The starting point of the simulation is the equilibrium HCP structure previously determined. Each atom was given an average initial kinetic energy corresponding to a temperature of $300 \mathrm{~K}$. Throughout the simulation the system was in contact with a heat bath at a constant temperature of $300 \mathrm{~K}$. In the top panel 
we depict the energy difference (per cage, in eV) between the actual configuration at time $t$ and the equilibrium configuration, where one can observe small oscillations around an average energy value reflecting the fact that the crystal is at finite temperature. In the lower panel we depict the time dependence of the deviation from the equilibrium value of the average cage radius (in percentage). Both numbers illustrate the small amplitude nature of the oscillations taking place [39].

Finally in Figure 10.11 we show the calculated band structures for the three bulk structures determined above. All three molecular solids are semiconductors

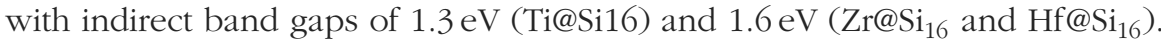

\subsection{Conclusions}

Making use of first principles computer simulations in the framework of DFT, we have investigated the main structural and electronic properties of the isovalent $X @ \mathrm{Si}_{16}$ ( $X=\mathrm{Ti}, \mathrm{Zr}$, Hf) nano-particles. We showed the feasibility of using these remarkably stable clusters to synthesize molecular solids and we characterized their main structural and electronic properties. Similar to bulk Ti@Si ${ }_{16}$,

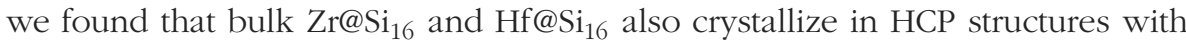
$\sim 4 \%$ larger inter-cage distance, compared to HCP-Ti@Si $i_{16}$. These bulk materials have a phase stability under isotropic compression up to $\sim 1 \mathrm{GPa}$ and bulk modulus also $\sim 1 \mathrm{GPa}$. Fully unconstrained QLMD simulations of the bulk structures suggest their stability at room temperature and normal pressure. Our

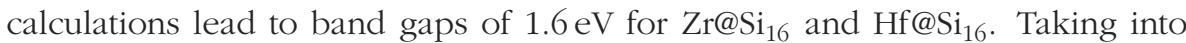
account that GGA systematically underestimates semi-conductor band gaps it is likely that the true band gap is larger than $2 \mathrm{eV}$.

The results obtained here suggest an interesting hierarchical rationale for the design of cluster assembled materials. Starting from the well known properties of the atoms, one can design target nanoparticles with pre-defined properties which, as such, are the constituent elements of new bulk materials. Furthermore, as shown here, when the nano-cage nucleates around a central atom, one can use at profit the size of the nucleating atom - via isovalent replacement - to 


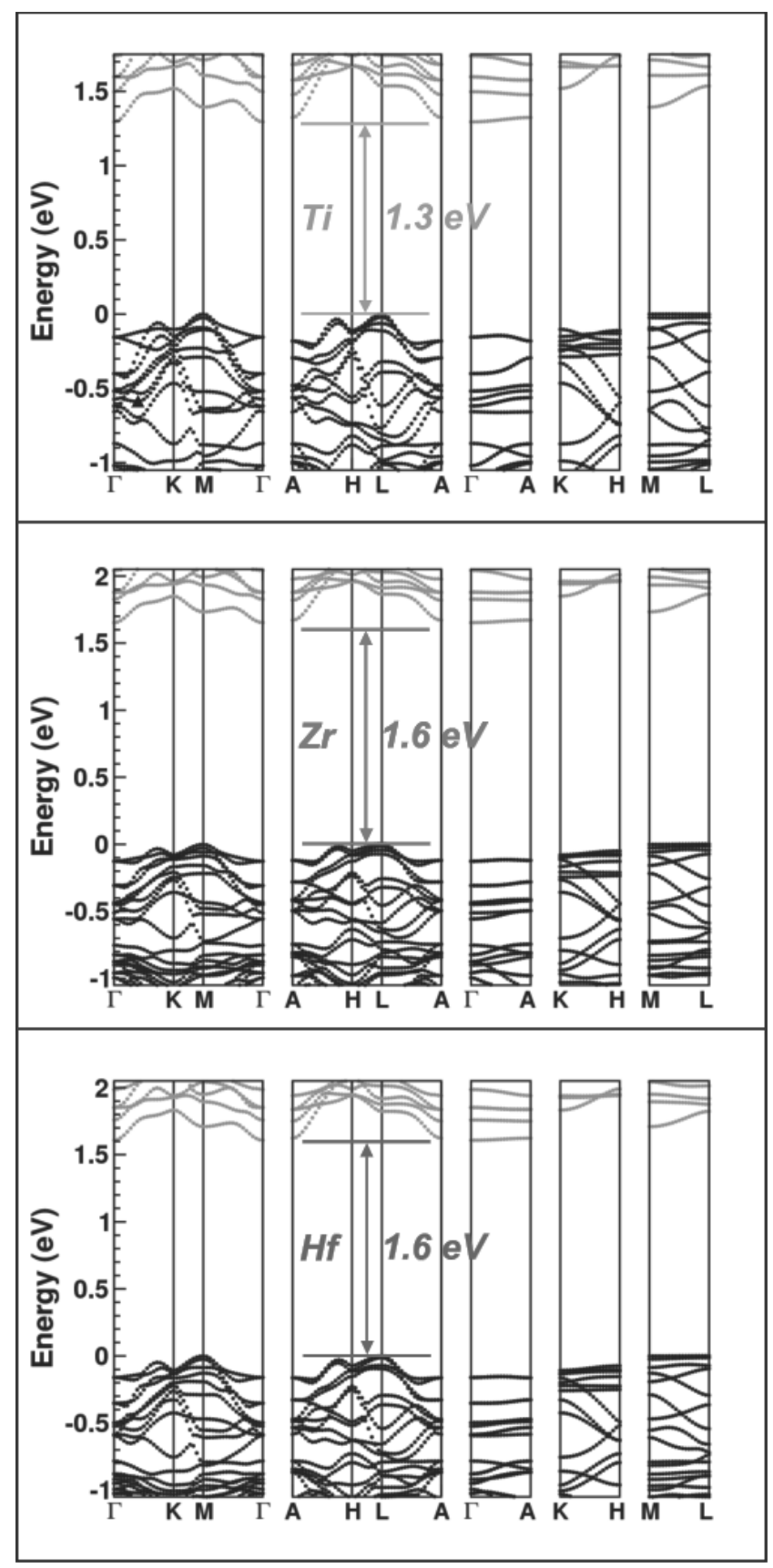

Figure 10.11. Calculated band structures of bulk $X @ S_{16}$ with $X=\mathrm{Ti}, \mathrm{Zr}$, Hf. These

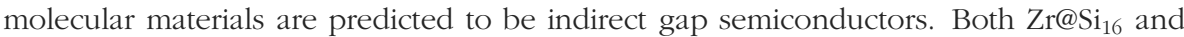
Hf@Si $i_{16}$ have larger band gaps than Ti@Si $i_{16}$. 
manipulate the cage size and, consequently, the bulk lattice, with direct implications on the band gap. This provides an additional degree of freedom which may prove very useful in, e.g., the quest for nano-designed, superconducting alloys. Taking fullerite as a model template, to the extent that doped bulk-X@Si ${ }_{16}$ is superconducting, changing the doping element and the nucleating nano-cage atom may provide additional laboratory knobs to tune the superconducting gap. The existence of low frequency intramolecular modes is another strong indicator that appropriately doped bulk-X@Si ${ }_{16}$ can exhibit a remarkable potential as possible high- $T_{C}$ superconductors. Ongoing calculations of the electron-phonon coupling to these nodes seem to support this. Additionally, the superconducting transition temperature of hypothetical doped bulk materials based on the $X @ S_{16}$ nanoparticles can be accurately predicted by means of computer simulations within the framework of the Migdal-Eliashberg [40-42] theory of phonon mediated superconductivity coupled to DFT. Work along these lines is in progress. We further hope that our results stimulate experiments aiming at synthesizing these materials in the lab $[13,15]$.

\section{Acknowledgments}

Financial support from FCT-Portugal is gratefully acknowledged.

\section{References}

[1] P. Hohenberg and W. Kohn, Phys. Rev. 136, B864 (1964).

[2] W. Kohn and L. J. Sham, Phys. Rev. 140, A1133 (1965).

[3] M. John, "Intel Says Chips Will Run Faster, Using Less Power" (New York Times, January 27, 2007).

[4] M. Schulz, Nature 399, 729 (1999).

[5] Clusters and Nanomaterials Springer Series in Cluster Physics (Springer, Heidelberg, 2002), edited by Y. Kawazoe, T. Kondow, and K. Ohno.

[6] S. M. Beck, J. Chem. Phys. 90, 6306 (1989); 87, 4233 (1987).

[7] S. M. Beck, Adv. Met. Semicond. Clusters 1, 241 (1993).

[8] H. Hiura, T. Miyazaki, and T. Kanayama, Phys. Rev. Lett. 86, 1733 (2001). 
[9] M. Ohara, K. Koyasu, A. Nakajima, and K. Kaya, Chem. Phys. Lett. 371, 490 (2003).

[10] K. Koyasu, M. Akutsu, M. Mitsui, and A. Nakajima, J. Am. Chem. Soc. 127, 4998 (2005).

[11] J. B. Jaeger, T. D. Jaeger, and M. A. Duncan, J. Phys. Chem. A 110, 9310 (2006).

[12] E. Janssens, P. Gruene, G. Meijer, L. Wste, P. Lievens, and A. Fielicke, Phys. Rev. Lett. 99, 063401 (2007).

[13] K. Koyasu, J. Atobe, M. Akutsu, M. Mitsui, and A. Nakajima, J. Phys. Chem. A 111, 42 (2007).

[14] S. Furuse, K. Koyasu, J. Atobe, and A. Nakajima, J. Chem. Phys. 129, 064311 (2008).

[15] J. T. Lau, K. Hirsch, Ph. Klar, A. Langenberg, F. Lofink, R. Richter, J. Rittmann, M. Vogel, V. Zamudio-Bayer, T. Möller, and B. v. Issendorff, Phys. Rev. A 79, 053201 (2009).

[16] V. Kumar and Y. Kawazoe, Phys. Rev. Lett. 87, 045503 (2001).

[17] V. Kumar and Y. Kawazoe, Phys. Rev. Lett. 88, 235504 (2002).

[18] J. Lu and S. Nagase, Phys. Rev. Lett. 90, 115506 (2003).

[19] J. U. Reveles and S. N. Khanna, Phys. Rev. B 74, 035435 (2006).

[20] P. Guo, Z. Y. Ren, A. P. Yang, J. G. Han, J. Bian, and G. H. Wang, J. Phys. Chem. A 110, 7453 (2006).

[21] M. B. Torres, E. M. Fernández, and L. C. Balbás, Phys. Rev. B 75, 205425 (2007).

[22] D. Bandyopadhyay, J. Appl. Phys. 104, 084308 (2008).

[23] G. K. Gueorguiev and J. M. Pacheco, J. Chem. Phys. 119, 10313 (2003).

[24] S. Zorriasatein, K. Joshi and D. G. Kanhere, Phys. Rev. B 75, 045117 (2007).

[25] J. M. Pacheco, G. K. Gueorguiev and J. L. Martins, Phys. Rev. B 66, 033401 (2002).

[26] C. L. Reis, J. L. Martins, and J. M. Pacheco, Phys. Rev. B 76, 233406 (2007)

[27] J. P. Perdew, K. Burke and M. Ernzerhof, Phys. Rev. Lett. 77, 3865 (1996).

[28] N. Troullier and J. L. Martins, Phys. Rev. B 43, 1993 (1991). 
[29] X. Gonze, J.-M. Beuken, R. Caracas, F. Detraux, M. Fuchs, G.-M. Rignanese, L. Sindic, M. Verstraete, G. Zerah, F. Jollet, M. Torrent, A. Roy, M. Mikami, P. Ghosez, J.-Y. Raty, and D. C. Allan, Comp. Mater. Sci. 25, 478 (2002).

[30] X. Gonze, G.-M. Rignanese, M. Verstraete, J.-M. Beuken, Y. Pouillon, R. Caracas, F. Jollet, M. Torrent, G. Zerah, M. Mikami, Ph. Ghosez, M. Veithen, J.-Y. Raty, V. Olevano, F. Bruneval, L. Reining, R. Godby, G. Onida, D. R. Hamann, and D. C. Allan, Zeit. Kristallogr. 220, 558 (2005).

[31] N. Binggeli, J. L. Martins, and J. R. Chelikowsky, Phys. Rev. Lett. 68, 2956 (1992).

[32] D. Porezag and M. Pederson, Phys. Rev. B 54, 7830 (1996).

[33] X. Gonze, Phys. Rev. B 55, 10337 (1997).

[34] X. Gonze and C. Lee, Phys. Rev. B 55, 10355 (1997).

[35] F. Birch, J. Geophys. Res. 57, 227 (1952).

[36] F. C. Frank and J. S. Kasper, Acta Crystallogr. 11, 184 (1958); 12, 483 (1959).

[37] S. Kugler, L. Pusztai, L. Rosta, P. Chieux, and R. Bellissent, Phys. Rev. B 48, $7685(1993)$

[38] N. Troullier and J. L. Martins, Phys. Rev. B 46, 1754 (1992).

[39] The very demanding nature of the computer simulations ultimately dictates the total QLMD simulation time. Besides the large energy cutoff of 30 Hartree required to ensure convergence of inter-atomic forces, no symmetry was enforced, such that both atomic coordinates and cell shape parameters were allowed to vary simultaneously. The total simulation time of 2 pico-second provides some evidence of the stability of this new molecular crystal. Nonetheless this does not fully warrant, however, its stability.

[40] G. M. Eliashberg, Sov. Phys. JETP 11, 696 (1960).

[41] G. M. Eliashberg, Zh. Eksp. Teor. Fiz. 38, 966 (1960).

[42] D. J. Scalapino, J. R. Schrieffer, and J. W. Wilkins, Phys. Rev. 148, 263 (1966). 